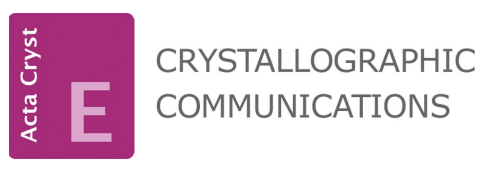

ISSN 2056-9890

Received 12 May 2018

Accepted 31 May 2018

Edited by D.-J. Xu, Zhejiang University (Yuquan Campus), China

Keywords: crystal structure; Schiff base; new crystal form; Hirshfeld surfaces; antimicrobial activity.

CCDC reference: 1846610

Supporting information: this article has supporting information at journals.iucr.org/e

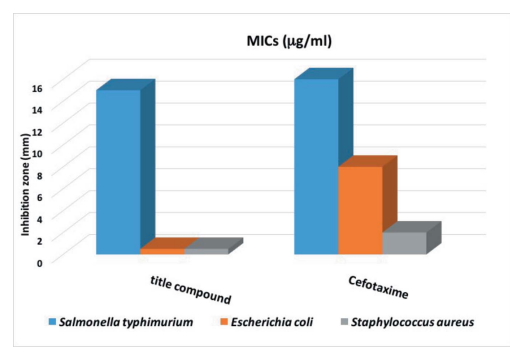

OPEN $\odot$ ACCESS

\section{A new crystal form and antimicrobial activity of (E)-1-[3-(2-hydroxybenzylideneamino)phenyl]- ethanone}

\author{
Nabila Moussa Slimane, Zakaria Bouhidel and Aouatef Cherouana*
}

Unité de Recherche de Chimie de I'Environnement et Moléculaire Structurale (URCHEMS), Département de Chimie, Université des freres Mentouri-Constantine 1, 25000 Constantine, Algeria. *Correspondence e-mail: c_aouatef@yahoo.fr

The title Schiff base compound, $\mathrm{C}_{15} \mathrm{H}_{13} \mathrm{NO}_{2}$, crystallizes in a new crystal form in the space group $P 22_{1} 2_{1} 2_{1}$, which is different from the monoclinic $P 2_{1} / n$ space group reported previously [De et al. (2009). Indian J. Chem. Sect. B, 48, 595598]. An intramolecular $\mathrm{O}-\mathrm{H} \cdots \mathrm{N}$ hydrogen bond occurs between the hydroxy and azomethine moieties. In the crystal, molecules are linked by weak $\mathrm{C}-$ $\mathrm{H}$. . O hydrogen bonds into supramolecular chains propagating along the $b$-axis direction with a $C(8)$ graph-set motif. The contribution of these two contacts in Hirshfeld surface area are around 19 and $21 \%$, respectively. The title compound was screened for its antibacterial activity against two gram-negative (Escherichia coli and Salmonella typhimurium) and one gram-positive (Staphyloccus aureus) bacteria. The results of this study reveal that this Schiff base shows good activity against only one bacterium, i.e. Salmonella typhimurium.

\section{Chemical context}

Schiff bases (Wang et al., 2008) are versatile ligands synthesized from the condensation of primary amines with carbonyl groups. These compounds have been shown to exhibit a broad range of biological activities, including antifungal, antibacterial, anti-malarial, antiproliferative, anti-inflammatory, antiviral and antipyretic properties (Dhar \& Taploo, 1982; Przybylski et al., 2009). Imine or azomethine groups are present in various natural, natural-derived and non-natural compounds and have been shown to be critical for their biological activity (Bringmann et al., 2004; de Souza et al., 2007).

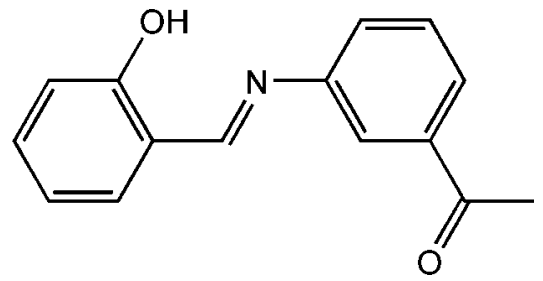

In this paper, we report the structural characterization using X-ray diffraction of the title Schiff base derived from salicylaldehyde, including an investigation of the Hirshfeld surfaces and its antimicrobial activity against two gram-negative (Escherichia coli and Salmonella typhimurium) and one grampositive (Staphyloccus aureus) bacteria. Schiff bases derived from this benzaldehyde are members of one of the most commonly investigated classes of compound, and have attracted the interest of chemists and physicists because they show photochromism and thermochromism in the solid state. 
Table 1

Selected geometric parameters $\left(\AA,^{\circ}\right)$.

\begin{tabular}{llll}
\hline $\mathrm{O} 1-\mathrm{C} 9$ & $1.349(3)$ & $\mathrm{N} 1-\mathrm{C} 1$ & $1.415(3)$ \\
$\mathrm{O} 2-\mathrm{C} 14$ & $1.213(3)$ & $\mathrm{N} 1-\mathrm{C} 7$ & $1.293(3)$ \\
$\mathrm{C} 1-\mathrm{N} 1-\mathrm{C} 7$ & $121.25(18)$ & $\mathrm{O} 1-\mathrm{C} 9-\mathrm{C} 10$ & $119.10(19)$ \\
$\mathrm{N} 1-\mathrm{C} 1-\mathrm{C} 2$ & $115.86(19)$ & $\mathrm{O} 1-\mathrm{C} 9-\mathrm{C} 8$ & $121.3(2)$ \\
$\mathrm{N} 1-\mathrm{C} 1-\mathrm{C} 6$ & $125.3(2)$ & $\mathrm{O} 2-\mathrm{C} 14-\mathrm{C} 15$ & $120.9(2)$ \\
$\mathrm{N} 1-\mathrm{C} 7-\mathrm{C} 8$ & $120.8(2)$ & $\mathrm{O} 2-\mathrm{C} 14-\mathrm{C} 3$ & $120.5(2)$ \\
\hline
\end{tabular}

Table 2

Hydrogen-bond geometry $\left(\AA{ }^{\circ}\right)$.

\begin{tabular}{lllll}
\hline$D-\mathrm{H} \cdots A$ & $D-\mathrm{H}$ & $\mathrm{H} \cdots A$ & $D \cdots A$ & $D-\mathrm{H} \cdots A$ \\
\hline $\mathrm{O} 1-\mathrm{H} 1 \cdots \mathrm{N} 1$ & 0.84 & 1.84 & $2.590(2)$ & 148 \\
$\mathrm{C} 7-\mathrm{H} 7 \cdots \mathrm{O} 2^{\mathrm{i}}$ & 0.95 & 2.40 & $3.321(3)$ & 162 \\
\hline
\end{tabular}

Symmetry code: (i) $-x+1, y-\frac{1}{2},-z+\frac{1}{2}$.

These photo- and thermochromic features are caused by proton transfer to the $\mathrm{N}$ atom from the $\mathrm{O}$ atom under the influence of light or temperature, respectively. It has been proposed that molecules showing thermochromism are planar and those showing photochromism are non-planar (Moustakali-Mavridis et al., 1980; Hadjoudis et al., 1987).

\section{Structural commentary}

The title Schiff base (Fig. 1) consists of two aromatic phenyl rings linked via an azomethine $(\mathrm{C}=\mathrm{N})$ group. The two phenyl rings are monosubstituted by a hydroxyl group on the same side as the azomethine carbon atom and by an aceto group on the other side. Relevant bond distances and angles are in good agreement with those reported in similar Schiff base compounds (Benarous et al., 2016; Chen et al., 2011). The C1$\mathrm{N} 1-\mathrm{C} 7-\mathrm{C} 8$ torsion angle of $-179.4(2)^{\circ}$ indicates an almost planar $E$ configuration with respect to the imine $\mathrm{C}=\mathrm{N}$ bond, as expected for a compound having an azomethine $\mathrm{HC}=\mathrm{N}$ bond as this is the most thermodynamically stable configuration (Ciciani et al., 2008). The $\mathrm{N} 1=\mathrm{C} 7[1.293$ (3) $\AA]$ and C9$\mathrm{C} 10[1.394$ (3) $\AA$ ] bond distances indicate that the compound adopts the phenol-imine tautomeric form with an $\mathrm{N}=\mathrm{C}$

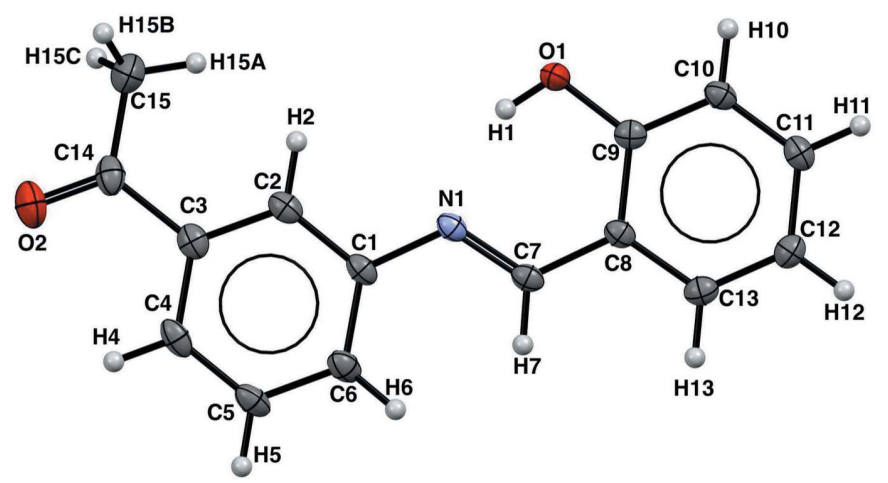

Figure 1

View of the title compound with the atom-numbering scheme. Displacement ellipsoids for non-H atoms are drawn at the $50 \%$ probability level.

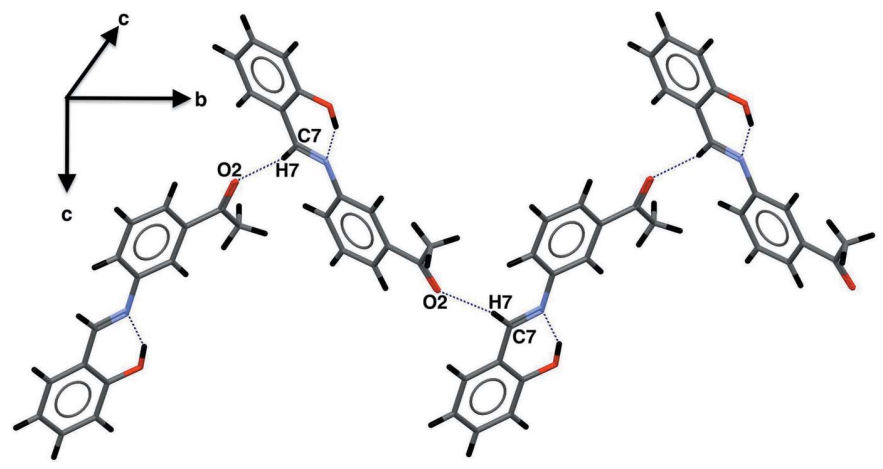

Figure 2

$\mathrm{C}-\mathrm{H} \cdots \mathrm{O}$ hydrogen bonds (Table 1 ) in the title compound.

double bond and a $\mathrm{C}-\mathrm{C}$ single bond (Table 1). Comparable values are observed in Schiff bases obtained from the same salicylaldehyde derivative with phenol-imine tautomeric form (Albayrak et al., 2010; Şahin et al., 2009).

An intramolecular $\mathrm{O}-\mathrm{H} \cdots \mathrm{N}$ hydrogen bond (Table 2) occurs between the O-hydroxyl and $\mathrm{N}$ azomethine atoms, forming an $S(6)$ ring motif. Such a hydrogen bond is frequently observed in Schiff bases derived from salicylaldehyde (Alpaslan et al., 2011).

\section{Supramolecular features}

In the crystal, the molecules are linked via $\mathrm{C}-\mathrm{H} \cdots \mathrm{O}$ hydrogen bonds between the carbon atom of azomethine group and the oxygen atom of the methoxy substituent, generating infinite chains with graph-set motif $C(8)$ along the $b$-axis direction (Table 2, Fig. 2). The chains are linked via $\pi-\pi$ interactions $(3.535 \AA$ ) between the $\mathrm{C} 8-\mathrm{C} 13$ benzene ring and the $\mathrm{C}=\mathrm{N}$ double bond (Fig. 3).

\section{Hirshfeld surfaces analysis}

Hirshfeld surfaces and two-dimensional fingerprint plots were generated by CrystalExplorer 3.1 (Wolff et al., 2012) to visualize and explore the intermolecular interactions. These molecular surfaces reflect intermolecular contacts based on colour coding distances from the surface to the nearest atom exterior $\left(d_{\mathrm{e}}\right)$ or interior $\left(d_{\mathrm{i}}\right)$ to the surface. In the Hirshfeld

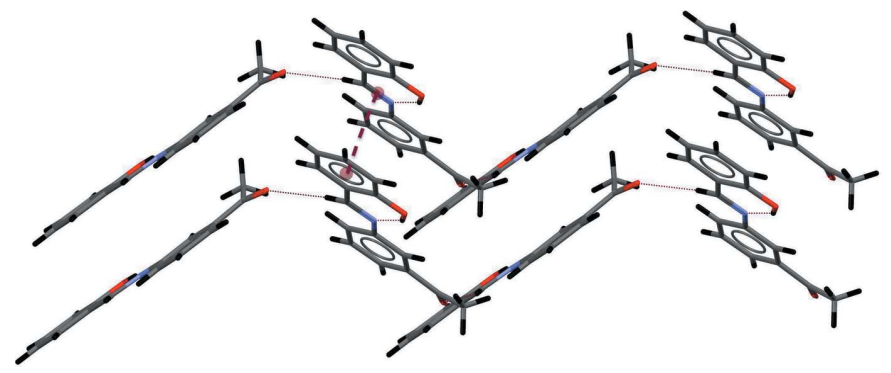

Figure 3

$\pi-\pi$ interaction between a benzene ring and the $\mathrm{C}=\mathrm{N}$ double bond. 


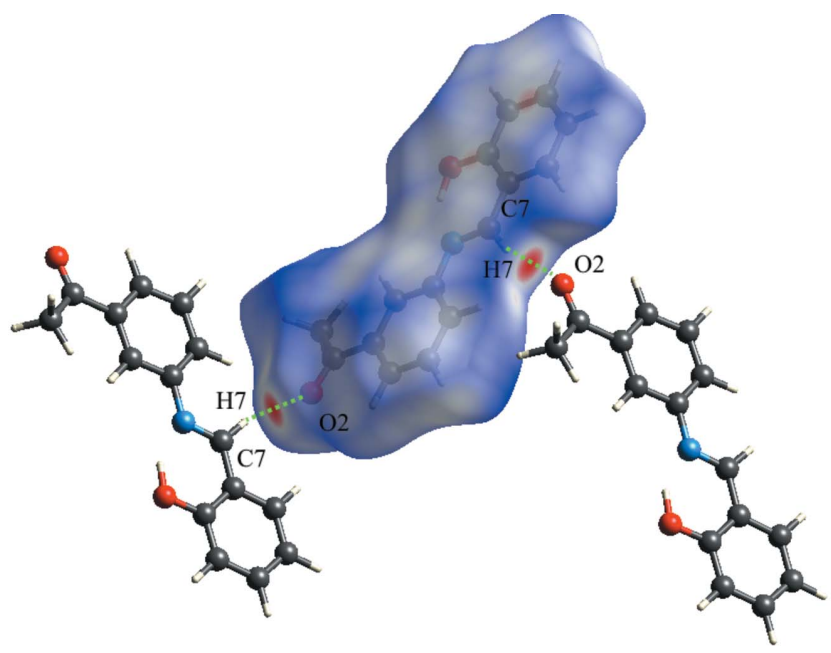

Figure 4

Hirshfeld surface of the title compound mapped over $d_{\text {norm }}(-0.60$ to 0.90 a.u.).

surface mapped over $d_{\text {norm }}$ (Fig. 4), red indicates the presence of short contacts and white represents contacts around the van der Waals separation, while the blue areas are completely devoid of close contacts. The intermolecular interactions were analysed by a combination of 3D Hirshfeld surfaces and 2D fingerprint plots, showing that the intermolecular $\mathrm{H} \cdots \mathrm{H}$ contacts make the largest contribution, corresponding to $46 \%$ of the total Hirshfeld surface area (Fig. 5). The presence of short intermolecular $\mathrm{H} \cdot \mathrm{H}$ contacts is observed in the vicinity of $2.30 \AA$. These contacts are manifested as white spots on the $d_{\text {norm }}$ surface and are considered to be weak interactions. In the fingerprint plots (Fig. 6), the $\mathrm{C} \cdot \mathrm{H} / \mathrm{H} \cdots \mathrm{C}$ contacts, representing $21.6 \%$ of the total Hirshfeld surface, appear as two short spikes. The red spots on the $d_{\text {norm }}$ surface in Fig. 4 are due to the $\mathrm{CH} \cdots \mathrm{O}$ contacts corresponding to the $\mathrm{C}-$ $\mathrm{H} \cdots \mathrm{O}$ hydrogen bond. The $\mathrm{O} \cdots \mathrm{H}$ contacts $(19.4 \%$ of the total Hirshfeld surface) show up as a sharp spike in the fingerprint plots at $d_{\mathrm{e}}+d_{\mathrm{i}} \simeq 2.3 \AA$. Finally, the packing cohesion in this structure is also provided by $\mathrm{C} \cdots \mathrm{N}$ and $\mathrm{C} \cdots \mathrm{C}$ interactions, which correspond to $\pi-\pi$ stacking interactions.

\section{Database survey}

A search for the title compound in the Cambridge Structural Database (Version 2.39; Groom et al., 2016) revealed that the crystal structure of the title compound had been previously been reported in the monoclinic $P 2_{1} / n$ space group (De et al., 2009). The latter differs from the title structure at position 3 of

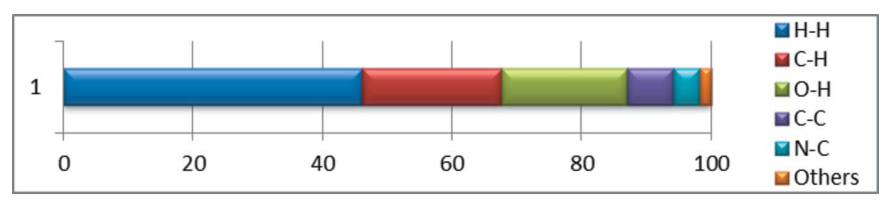

Figure 5

Relative contributions of various interactions to the Hirshfeld surface area.
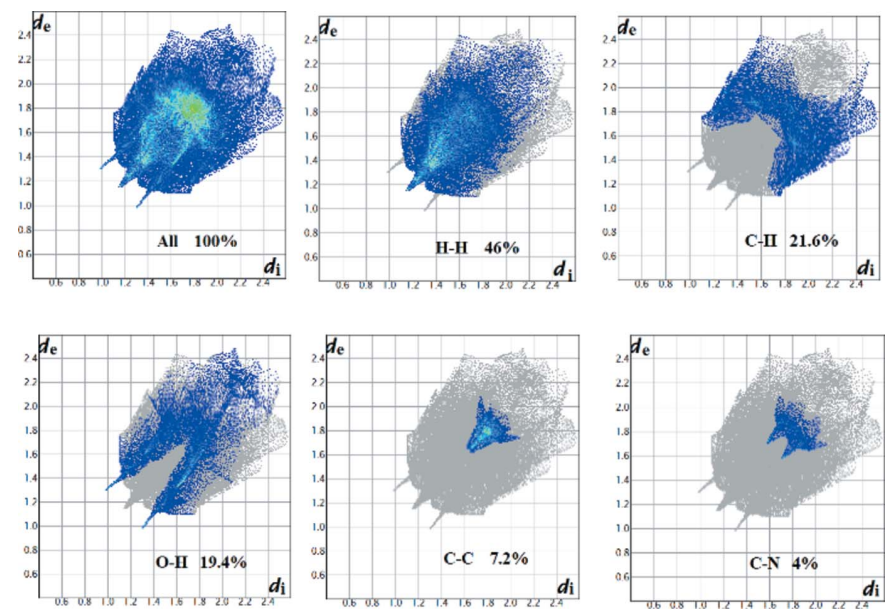

Figure 6

Two-dimensional fingerprints of the compound, showing all interactions and $\mathrm{H} \cdots \mathrm{H}, \mathrm{C} \cdots \mathrm{H}, \mathrm{O} \cdots \mathrm{H}, \mathrm{C} \cdots \mathrm{C}$ and $\mathrm{C} \cdots \mathrm{N}$ contacts.

the aceto substituent, which is on the same side of the hydroxyl group in the title compound and in the opposite side in the reported one. This difference in position directly affects the hydrogen-bonding pattern. Similar infinite $\mathrm{C}-\mathrm{H} \cdots \mathrm{O}$ chains occur in both compounds but the angle between linked molecules is $c a 67.99^{\circ}$ in the title compound and $77.61^{\circ}$ in the reported one. The CSD search also found seven hits for structures containing the title molecule but with additional substituents (a methoxy or an additional hydroxyl group).

\section{Antimicrobial activity}

The title compound was screened for its antibacterial activity against two gram-negative (Escherichia coli and Salmonella typhimurium) and one gram-positive (Staphyloccus aureus) bacterial strains by the agar-well diffusion method (Cruickshank,1970). The solvent DMSO was used as negative control. A $0.5 \mathrm{ml}$ spore suspension $\left(10^{-6}-10^{-7}\right.$ spore $\left.\mathrm{ml}^{-1}\right)$ of each of

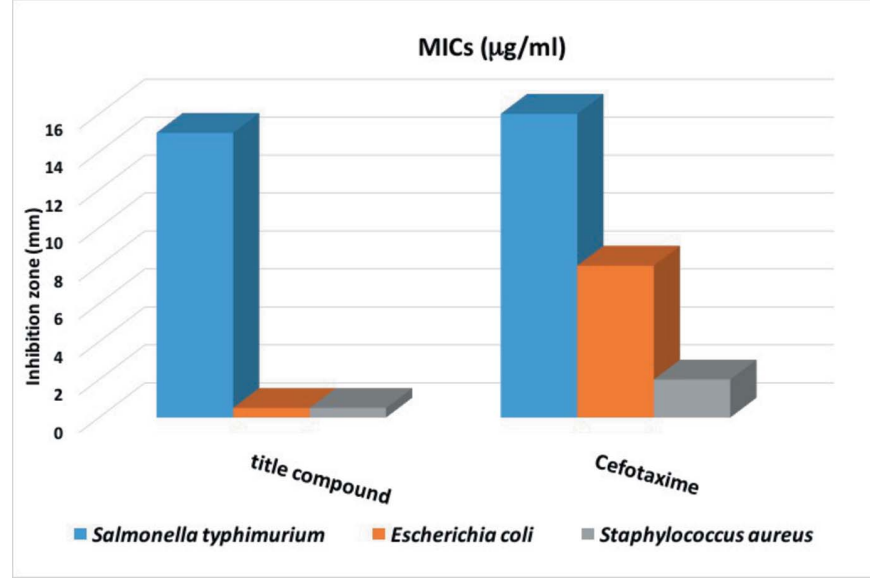

Figure 7

MICs (minimum inhibitory concentrations) antibacterial activity of the title compound and the standard Cefotaxime 
Table 3

Experimental details.

\begin{tabular}{|c|c|}
\hline \multicolumn{2}{|l|}{ Crystal data } \\
\hline Chemical formula & $\mathrm{C}_{15} \mathrm{H}_{13} \mathrm{NO}_{2}$ \\
\hline$M_{\mathrm{r}}$ & 239.26 \\
\hline Crystal system, space group & Orthorhombic, $P 2_{1} 2_{1} 2_{1}$ \\
\hline Temperature (K) & 100 \\
\hline$a, b, c(\AA)$ & $\begin{array}{l}4.8637(3), 14.6601(10), \\
\quad 16.6512(9)\end{array}$ \\
\hline$V\left(\AA^{3}\right)$ & $1187.27(13)$ \\
\hline$Z$ & 4 \\
\hline Radiation type & $\mathrm{Cu} K \alpha$ \\
\hline$\mu\left(\mathrm{mm}^{-1}\right)$ & 0.72 \\
\hline Crystal size $(\mathrm{mm})$ & $0.1 \times 0.1 \times 0.08$ \\
\hline \multicolumn{2}{|l|}{ Data collection } \\
\hline Diffractometer & $\begin{array}{l}\text { Oxford Diffraction Xcalibur } \\
\text { Sapphire2 CCD }\end{array}$ \\
\hline Absorption correction & $\begin{array}{l}\text { Integration (ABSORB; DeTitta, } \\
\text { 1985) }\end{array}$ \\
\hline$T_{\min }, T_{\max }$ & $0.966,0.991$ \\
\hline $\begin{array}{l}\text { No. of measured, independent and } \\
\text { observed }[I>2 \sigma(I)] \text { reflections }\end{array}$ & $10738,2461,2222$ \\
\hline$R_{\text {int }}$ & 0.058 \\
\hline$(\sin \theta / \lambda)_{\max }\left(\AA^{-1}\right)$ & 0.632 \\
\hline \multicolumn{2}{|l|}{ Refinement } \\
\hline$R\left[F^{2}>2 \sigma\left(F^{2}\right)\right], w R\left(F^{2}\right), S$ & $0.046,0.131,1.07$ \\
\hline No. of reflections & 2461 \\
\hline No. of parameters & 163 \\
\hline $\mathrm{H}$-atom treatment & $\mathrm{H}$-atom parameters constrained \\
\hline$\Delta \rho_{\max }, \Delta \rho_{\min }\left(\mathrm{e} \AA^{-3}\right)$ & $0.33,-0.36$ \\
\hline Absolute structure & $\begin{array}{l}\text { Flack } x \text { determined using } 838 \\
\quad \text { quotients }\left[\left(I^{+}\right)-\left(I^{-}\right)\right] /\left[\left(I^{+}\right)+\left(I^{-}\right)\right] \\
\quad \text { (Parsons } \text { et al., } 2013)\end{array}$ \\
\hline Absolute structure parameter & $0.00(19)$ \\
\hline
\end{tabular}

Computer programs: CrysAlis PRO (Rigaku OD, 2015), SIR2004 (Burla et al., 2005), SHELXL (Sheldrick, 2015) and OLEX2 (Dolomanov et al., 2009).

the investigated organisms was added to a sterile agar medium just before solidification, then poured into sterile petri dishes (9 $\mathrm{cm}$ in diameter) and left to solidify. Using a sterile cork borer ( $6 \mathrm{~mm}$ in diameter), five holes (wells) were made in each dish, and then $5 \mu \mathrm{L}$ of the tested compound, dissolved in DMSO with different concentrations $(\mathrm{C}, \mathrm{C} / 2, \mathrm{C} / 4, \mathrm{C} / 8)$, was poured into these holes. Finally, the dishes were incubated at $310 \mathrm{~K}$ for $48 \mathrm{~h}$. Clear or inhibition zones were detected around each hole. DMSO alone $(0.5 \mu \mathrm{L})$ was used as a control under the same conditions for each organism by subtracting the diameter of inhibition zone resulting with DMSO from that obtained in the study compound. The antibacterial activity of Cefotaxime was also measured in comparison to the title compound and used as a standard to reveal the potency of synthesized derivative.

The results of the antimicrobial screening indicate that the compound shows significant activity only against Salmonella typhimurium with an inhibition zone diameter of $15 \mathrm{~mm}$. This value is close to that observed with the standard used (Cefotaxime) against the same bacterium $(16 \mathrm{~mm})$. For the two other bacteria, Escherichia coli and Staphyloccus aureus, the standard exhibits a higher activity than the study compound, for which the inhibition zone diameter is under $2 \mathrm{~mm}$. These results are summarized in Fig. 7, which gives the MICs (minimum inhibitory concentrations) of the title Schiff base and Cefotaxime.

\section{Synthesis and crystallization}

The title Schiff base was synthesized by reacting 3-aminoacetophenone $(0.13 \mathrm{~g}, 1 \mathrm{mmol})$ and salicylaldehyde $(0.12 \mathrm{~g}$, $1 \mathrm{mmol})$ in ethanol $(20 \mathrm{ml})$. The resulting mixture was refluxed for $3 \mathrm{~h}$. Yellow single crystals suitable for single crystal X-ray diffraction studies were obtained by slow evaporation of the solution.

\section{Refinement}

Crystal data, data collection and structure refinement details are summarized in Table 3. All $\mathrm{H}$ atoms were located in difference electron-density maps and treated as riding on their parent atoms, with $\mathrm{C}-\mathrm{H}=0.95-0.98 \AA$ with $U_{\text {iso }}(\mathrm{H})=$ $1.2 U_{\text {eq }}(\mathrm{C})$ or $1.5 U_{\text {eq }}($ Cmethyl $)$ and $\mathrm{O}-\mathrm{H}=0.84 \AA$ with $U_{\text {iso }}(\mathrm{H})=1.5 U_{\text {eq }}(\mathrm{O})$.

\section{Acknowledgements}

The authors acknowledge CRM2, Institut Jean Barriol (UMR 7036 CNRS, University de Lorraine, France), for providing access to the experimental crystallographic facilities. Dr Slimane Dahaoui and Dr Emmanuel Wenger are thanked for their help in collectiong diffraction data, Professor Foudil Khelifa, Director of the Laboratoire d'hygiène de la Wilaya de Constantine, Algeria, for use of the antimicrobial facility and biologists Zine Faiza, Haifi Maya and Belhaffaf Mouni for their help.

\section{References}

Albayrak, Ç., Koşar, B., Demir, S., Odabaşoğlu, M. \& Büyükgüngör, O. (2010). J. Mol. Struct. 963, 211-218.

Alpaslan, Y. B., Alpaslan, G., Ağar, A. A., İskeleli, N. O. \& Öztekin, E. (2011). J. Mol. Struct. 995, 58-65.

Benarous, N., Cherouana, A., Aubert, E., Durand, P. \& Dahaoui, S. (2016). J. Mol. Struct. 1105, 186-193.

Bringmann, G., Dreyer, M., Faber, J. H., Dalsgaard, P. W., Staerk, D., Jaroszewski, J. W., Ndangalasi, H., Mbago, F., Brun, R. \& Christensen, S. B. (2004). J. Nat. Prod. 67, 743-748.

Burla, M. C., Caliandro, R., Camalli, M., Carrozzini, B., Cascarano, G. L., De Caro, L., Giacovazzo, C., Polidori, G. \& Spagna, R. (2005). J. Appl. Cryst. 38, 381-388.

Chen, X.-T., Xiang, Y., Song, P.-S., Wei, R., Zhou, Z.-J., Li, K. \& Tong, A.-J. (2011). J. Lumin. 131, 1453-1459.

Ciciani, G., Coronnello, M., Guerrini, G., Selleri, S., Cantore, M., Failli, P., Mini, E. \& Costanzo, A. (2008). Bioorg. Med. Chem. 16, 9409-9419.

Cruickshank, R. (1970). Medical Microbiology, 11th ed., pp. 652, 901. Edinburgh and London: E \& S. Livingstone Ltd.

De, R. L., Mukherjee, J., Mandal, M., Roy, L., Bhowal, R. \& Banerjee, I. (2009). Indian J. Chem. Sect B, 48, 595-598.

DeTitta, G. T. (1985). J. Appl. Cryst. 18, 75-79.

Dhar, D. N. \& Taploo, C. L. (1982). J. Sci. Ind. Res. 41, 501-506.

Dolomanov, O. V., Bourhis, L. J., Gildea, R. J., Howard, J. A. K. \& Puschmann, H. (2009). J. Appl. Cryst. 42, 339-341.

Groom, C. R., Bruno, I. J., Lightfoot, M. P. \& Ward, S. C. (2016). Acta Cryst. B72, 171-179.

Hadjoudis, E., Vittorakis, M. \& Moustakali-Mavridis, I. (1987). Tetrahedron, 43, 1345-1360.

Moustakali-Mavridis, I., Hadjoudis, B. \& Mavridis, A. (1980). Acta Cryst. B36, 1126-1130. 
Parsons, S., Flack, H. D. \& Wagner, T. (2013). Acta Cryst. B69, 249259.

Przybylski, P., Huczynski, A., Pyta, K., Brzezinski, B. \& Bartl, F. (2009). Curr. Org. Chem. 13, 124-148.

Rigaku OD (2015). CrysAlis PRO. Rigaku Oxford Diffraction, Yarnton, England.

Şahin, Z. S., Gūmūş, S., Macit, M. \& Işık, Ş. (2009). Acta Cryst. E65, o3022.
Sheldrick, G. M. (2015). Acta Cryst. C71, 3-8.

Souza, A. O. de, Galetti, F. C. S., Silva, C. L., Bicalho, B., Parma, M. M. \& Fonseca, S. F. (2007). Quim Nova, 30, 1563-1566.

Wang, L., Feng, Y., Xue, J. \& Yukun, L. (2008). J. Serb. Chem. Soc. 73, $1-6$.

Wolff, S. K., Grimwood, D. J., McKinnon, J. J., Turner, M. J., Jayatilaka, D. \& Spackman, M. A. (2012). CrystalExplorer. The University of Western Australia. 


\section{supporting information}

Acta Cryst. (2018). E74, 884-888 [https://doi.org/10.1107/S205698901800806X]

\section{A new crystal form and antimicrobial activity of (E)-1-[3-(2-hydroxybenzyl- ideneamino)phenyl]ethanone}

\section{Nabila Moussa Slimane, Zakaria Bouhidel and Aouatef Cherouana}

Computing details

Data collection: CrysAlis PRO (Rigaku OD, 2015); cell refinement: CrysAlis PRO (Rigaku OD, 2015); data reduction: CrysAlis PRO (Rigaku OD, 2015); program(s) used to solve structure: SIR2004 (Burla et al., 2005); program(s) used to refine structure: SHELXL (Sheldrick, 2015); molecular graphics: OLEX2 (Dolomanov et al., 2009); software used to prepare material for publication: OLEX2 (Dolomanov et al., 2009).

(E)-1-[3-(2-Hydroxybenzylideneamino) phenyl] ethanone

\section{Crystal data}

$\mathrm{C}_{15} \mathrm{H}_{13} \mathrm{NO}_{2}$

$M_{r}=239.26$

Orthorhombic, $P 2{ }_{1} 2_{1} 2_{1}$

Hall symbol: P 2ac 2ab

$a=4.8637(3) \AA$

$b=14.6601(10) \AA$

$c=16.6512(9) \AA$

$V=1187.27(13) \AA^{3}$

$Z=4$

\section{Data collection}

Oxford Diffraction Xcalibur Sapphire2 CCD diffractometer

Radiation source: fine-focus sealed tube Graphite monochromator $\varphi$ and $\omega$ scans

Absorption correction: integration (ABSORB; DeTitta, 1985)

$T_{\min }=0.966, T_{\max }=0.991$

\section{Refinement}

Refinement on $F^{2}$

Least-squares matrix: full

$R\left[F^{2}>2 \sigma\left(F^{2}\right)\right]=0.046$

$w R\left(F^{2}\right)=0.131$

$S=1.07$

2461 reflections

163 parameters

0 restraints

$\mathrm{H}$-atom parameters constrained
$F(000)=504$

$D_{\mathrm{x}}=1.339 \mathrm{Mg} \mathrm{m}^{-3}$

$\mathrm{Cu} K \alpha$ radiation, $\lambda=1.54184 \AA$

Cell parameters from 10738 reflections

$\theta=4.0-77.0^{\circ}$

$\mu=0.72 \mathrm{~mm}^{-1}$

$T=100 \mathrm{~K}$

Prism, yellow

$0.1 \times 0.1 \times 0.08 \mathrm{~mm}$

10738 measured reflections

2461 independent reflections

2222 reflections with $I>2 \sigma(I)$

$R_{\text {int }}=0.058$

$\theta_{\max }=77.0^{\circ}, \theta_{\min }=4.0^{\circ}$

$h=-5 \rightarrow 6$

$k=-18 \rightarrow 18$

$l=-20 \rightarrow 20$

$w=1 /\left[\sigma^{2}\left(F_{\mathrm{o}}{ }^{2}\right)+(0.0633 P)^{2}+0.6475 P\right]$ where $P=\left(F_{\mathrm{o}}^{2}+2 F_{\mathrm{c}}{ }^{2}\right) / 3$

$(\Delta / \sigma)_{\max }<0.001$

$\Delta \rho_{\max }=0.33 \mathrm{e} \AA^{-3}$

$\Delta \rho_{\min }=-0.36$ e $\AA^{-3}$

Absolute structure: Flack $x$ determined using 838 quotients $\left[\left(I^{+}\right)-\left(I^{)}\right)\right] /\left[\left(I^{+}\right)+\left(I^{-}\right)\right]$(Parsons et al., 2013)

Absolute structure parameter: 0.00 (19) 


\section{Special details}

Geometry. Bond distances, angles etc. have been calculated using the rounded fractional coordinates. All su's are estimated from the variances of the (full) variance-covariance matrix. The cell esds are taken into account in the estimation of distances, angles and torsion angles

Fractional atomic coordinates and isotropic or equivalent isotropic displacement parameters $\left(\AA^{2}\right)$

\begin{tabular}{|c|c|c|c|c|}
\hline & $x$ & $y$ & $z$ & $U_{\text {iso }} * / U_{\text {eq }}$ \\
\hline $\mathrm{O} 1$ & $-0.2016(4)$ & $0.05562(11)$ & $0.50631(9)$ & 0.0218 \\
\hline $\mathrm{O} 2$ & $0.9481(4)$ & $0.29985(14)$ & $0.27572(12)$ & $0.0356(6)$ \\
\hline N1 & $0.0723(4)$ & $0.03478(13)$ & $0.37418(10)$ & $0.0173(5)$ \\
\hline $\mathrm{C} 1$ & $0.2784(5)$ & $0.06549(15)$ & $0.32063(12)$ & $0.0175(6)$ \\
\hline $\mathrm{C} 2$ & $0.4217(5)$ & $0.14349(16)$ & $0.34432(13)$ & $0.0202(6)$ \\
\hline $\mathrm{C} 3$ & $0.6303(5)$ & $0.17989(15)$ & $0.29616(13)$ & $0.0201(6)$ \\
\hline $\mathrm{C} 4$ & $0.6939(5)$ & $0.13896(17)$ & $0.22293(13)$ & $0.0225(6)$ \\
\hline C5 & $0.5530(5)$ & $0.06016(17)$ & $0.19957(13)$ & $0.0233(6)$ \\
\hline $\mathrm{C} 6$ & $0.3462(5)$ & $0.02425(17)$ & $0.24763(13)$ & $0.0225(7)$ \\
\hline $\mathrm{C} 7$ & $-0.0656(5)$ & $-0.03891(15)$ & $0.36008(13)$ & $0.0183(6)$ \\
\hline $\mathrm{C} 8$ & $-0.2785(5)$ & $-0.06931(15)$ & $0.41500(13)$ & $0.0173(6)$ \\
\hline C9 & $-0.3394(5)$ & -0.02099 (14) & $0.48639(12)$ & $0.0174(6)$ \\
\hline $\mathrm{C} 10$ & $-0.5447(5)$ & $-0.05303(15)$ & $0.53750(13)$ & $0.0196(6)$ \\
\hline $\mathrm{C} 11$ & $-0.6896(5)$ & $-0.13149(15)$ & $0.51850(13)$ & $0.0203(6)$ \\
\hline $\mathrm{C} 12$ & $-0.6348(5)$ & $-0.17920(15)$ & $0.44765(14)$ & $0.0208(6)$ \\
\hline $\mathrm{C} 13$ & $-0.4307(5)$ & $-0.14870(15)$ & $0.39705(13)$ & $0.0199(6)$ \\
\hline $\mathrm{C} 14$ & $0.7915(5)$ & $0.26259(16)$ & $0.32198(15)$ & $0.0243(7)$ \\
\hline $\mathrm{C} 15$ & $0.7613(6)$ & $0.29571(19)$ & $0.40716(18)$ & $0.0354(8)$ \\
\hline $\mathrm{H} 1$ & -0.08597 & 0.06773 & 0.47038 & $0.0326^{*}$ \\
\hline $\mathrm{H} 2$ & 0.37706 & 0.17221 & 0.39377 & $0.0242 *$ \\
\hline $\mathrm{H} 4$ & 0.83170 & 0.16435 & 0.18922 & $0.0270^{*}$ \\
\hline H5 & 0.59906 & 0.03096 & 0.15045 & $0.0280^{*}$ \\
\hline H6 & 0.25006 & -0.02878 & 0.23072 & $0.0270^{*}$ \\
\hline $\mathrm{H} 7$ & -0.02712 & -0.07358 & 0.31321 & $0.0219 *$ \\
\hline $\mathrm{H} 10$ & -0.58548 & -0.02092 & 0.58561 & $0.0235^{*}$ \\
\hline H11 & -0.82777 & -0.15309 & 0.55406 & $0.0244 *$ \\
\hline $\mathrm{H} 12$ & -0.73747 & -0.23226 & 0.43451 & $0.0250 *$ \\
\hline $\mathrm{H} 13$ & -0.39171 & -0.18163 & 0.34925 & $0.0239 *$ \\
\hline $\mathrm{H} 15 \mathrm{~A}$ & 0.62683 & 0.25763 & 0.43540 & $0.0531 *$ \\
\hline H15B & 0.69811 & 0.35919 & 0.40695 & $0.0531^{*}$ \\
\hline $\mathrm{H} 15 \mathrm{C}$ & 0.93926 & 0.29186 & 0.43454 & $0.0531 *$ \\
\hline
\end{tabular}

Atomic displacement parameters $\left(\AA^{2}\right)$

\begin{tabular}{lllllll}
\hline & $U^{11}$ & $U^{22}$ & $U^{33}$ & $U^{12}$ & $U^{13}$ & $U^{23}$ \\
\hline O1 & $0.0218(8)$ & $0.0262(8)$ & $0.0173(7)$ & $-0.0043(6)$ & $0.0034(6)$ & $-0.0041(6)$ \\
O2 & $0.0345(11)$ & $0.0348(10)$ & $0.0376(10)$ & $-0.0070(8)$ & $0.0067(9)$ & $0.0108(8)$ \\
N1 & $0.0156(9)$ & $0.0224(9)$ & $0.0140(8)$ & $0.0023(7)$ & $0.0002(7)$ & $0.0011(7)$ \\
C1 & $0.0160(10)$ & $0.0225(10)$ & $0.0141(10)$ & $0.0040(9)$ & $0.0006(8)$ & $0.0045(8)$ \\
C2 & $0.0193(11)$ & $0.0239(11)$ & $0.0173(10)$ & $0.0052(9)$ & $0.0013(9)$ & $0.0024(8)$
\end{tabular}




\begin{tabular}{lllllll} 
C3 & $0.0181(12)$ & $0.0220(10)$ & $0.0202(10)$ & $0.0032(8)$ & $-0.0003(8)$ & $0.0057(8)$ \\
C4 & $0.0174(11)$ & $0.0333(12)$ & $0.0169(10)$ & $0.0035(10)$ & $0.0011(9)$ & $0.0103(9)$ \\
C5 & $0.0212(12)$ & $0.0349(12)$ & $0.0139(9)$ & $0.0035(10)$ & $0.0016(9)$ & $0.0015(9)$ \\
C6 & $0.0182(12)$ & $0.0337(12)$ & $0.0155(10)$ & $-0.0008(10)$ & $-0.0008(8)$ & $0.0002(9)$ \\
C7 & $0.0196(11)$ & $0.0217(10)$ & $0.0135(9)$ & $0.0035(9)$ & $-0.0005(9)$ & $0.0011(8)$ \\
C8 & $0.0173(11)$ & $0.0206(10)$ & $0.0141(10)$ & $0.0021(8)$ & $-0.0011(8)$ & $0.0014(8)$ \\
C9 & $0.0183(11)$ & $0.0188(9)$ & $0.0151(9)$ & $0.0027(8)$ & $-0.0033(8)$ & $0.0015(8)$ \\
C10 & $0.0201(11)$ & $0.0239(10)$ & $0.0147(9)$ & $0.0016(9)$ & $0.0001(8)$ & $-0.0002(8)$ \\
C11 & $0.0175(10)$ & $0.0242(10)$ & $0.0193(10)$ & $0.0014(9)$ & $0.0014(9)$ & $0.0043(8)$ \\
C12 & $0.0207(12)$ & $0.0183(10)$ & $0.0235(11)$ & $-0.0008(8)$ & $-0.0030(9)$ & $0.0008(8)$ \\
C13 & $0.0220(12)$ & $0.0193(10)$ & $0.0185(10)$ & $0.0021(9)$ & $-0.0034(9)$ & $-0.0006(8)$ \\
C14 & $0.0210(12)$ & $0.0200(10)$ & $0.0320(12)$ & $0.0013(9)$ & $0.0027(10)$ & $0.0066(9)$ \\
C15 & $0.0385(16)$ & $0.0290(12)$ & $0.0388(15)$ & $-0.0107(11)$ & $0.0066(12)$ & $-0.0038(11)$ \\
\hline
\end{tabular}

Geometric parameters $\left(\AA,{ }^{\circ}\right)$

\begin{tabular}{|c|c|c|c|}
\hline $\mathrm{O} 1-\mathrm{C} 9$ & $1.349(3)$ & $\mathrm{C} 10-\mathrm{C} 11$ & $1.386(3)$ \\
\hline $\mathrm{O} 2-\mathrm{C} 14$ & $1.213(3)$ & $\mathrm{C} 11-\mathrm{C} 12$ & $1.397(3)$ \\
\hline $\mathrm{O} 1-\mathrm{H} 1$ & 0.8400 & $\mathrm{C} 12-\mathrm{C} 13$ & $1.377(3)$ \\
\hline $\mathrm{N} 1-\mathrm{C} 1$ & $1.415(3)$ & $\mathrm{C} 14-\mathrm{C} 15$ & $1.506(4)$ \\
\hline $\mathrm{N} 1-\mathrm{C} 7$ & $1.293(3)$ & $\mathrm{C} 2-\mathrm{H} 2$ & 0.9500 \\
\hline $\mathrm{C} 1-\mathrm{C} 6$ & $1.397(3)$ & $\mathrm{C} 4-\mathrm{H} 4$ & 0.9500 \\
\hline $\mathrm{C} 1-\mathrm{C} 2$ & $1.396(3)$ & $\mathrm{C} 5-\mathrm{H} 5$ & 0.9500 \\
\hline $\mathrm{C} 2-\mathrm{C} 3$ & $1.399(3)$ & C6- $-\mathrm{H} 6$ & 0.9500 \\
\hline $\mathrm{C} 3-\mathrm{C} 4$ & $1.394(3)$ & $\mathrm{C} 7-\mathrm{H} 7$ & 0.9500 \\
\hline $\mathrm{C} 3-\mathrm{C} 14$ & $1.507(3)$ & $\mathrm{C} 10-\mathrm{H} 10$ & 0.9500 \\
\hline $\mathrm{C} 4-\mathrm{C} 5$ & $1.398(3)$ & $\mathrm{C} 11-\mathrm{H} 11$ & 0.9500 \\
\hline $\mathrm{C} 5-\mathrm{C} 6$ & $1.389(3)$ & $\mathrm{C} 12-\mathrm{H} 12$ & 0.9500 \\
\hline $\mathrm{C} 7-\mathrm{C} 8$ & $1.452(3)$ & $\mathrm{C} 13-\mathrm{H} 13$ & 0.9500 \\
\hline $\mathrm{C} 8-\mathrm{C} 13$ & $1.411(3)$ & $\mathrm{C} 15-\mathrm{H} 15 \mathrm{~A}$ & 0.9800 \\
\hline $\mathrm{C} 8-\mathrm{C} 9$ & $1.415(3)$ & C15-H15B & 0.9800 \\
\hline $\mathrm{C} 9-\mathrm{C} 10$ & $1.394(3)$ & $\mathrm{C} 15-\mathrm{H} 15 \mathrm{C}$ & 0.9800 \\
\hline $\mathrm{O} 1 \cdots \mathrm{N} 1$ & $2.590(2)$ & $\mathrm{C} 5 \cdots \mathrm{H} 11^{\mathrm{ix}}$ & 2.9900 \\
\hline $\mathrm{O} 2 \cdots \mathrm{C}^{\mathrm{i}}$ & $3.321(3)$ & $\mathrm{C} 5 \cdots \mathrm{H} 10^{\mathrm{ix}}$ & 3.0200 \\
\hline $\mathrm{O} 1 \cdots \mathrm{H} 15 \mathrm{~B}^{\mathrm{ii}}$ & 2.7200 & $\mathrm{C} 6 \cdots \mathrm{H} 10^{\text {ix }}$ & 2.9800 \\
\hline $\mathrm{O} 1 \cdots \mathrm{H} 5^{\mathrm{iii}}$ & 2.7600 & $\mathrm{C} 6 \cdots \mathrm{H} 7$ & 2.5600 \\
\hline $\mathrm{O} 2 \cdots \mathrm{H} 4$ & 2.5200 & $\mathrm{C} 7 \cdots \mathrm{H} 6$ & 2.6500 \\
\hline $\mathrm{O} 2 \cdots \mathrm{H} 6^{\mathrm{i}}$ & 2.6900 & $\mathrm{C} 7 \cdots \mathrm{H} 1$ & 2.4100 \\
\hline $\mathrm{O} 2 \cdots \mathrm{H} 7^{\mathrm{i}}$ & 2.4000 & $\mathrm{C} 9 \cdots \mathrm{H} 5^{\mathrm{iii}}$ & 2.9700 \\
\hline $\mathrm{N} 1 \cdots \mathrm{O} 1$ & $2.590(2)$ & $\mathrm{C} 10 \cdots \mathrm{H} 5^{\mathrm{iii}}$ & 2.8900 \\
\hline $\mathrm{N} 1 \cdots \mathrm{C} 3^{\text {iv }}$ & $3.292(3)$ & $\mathrm{C} 11 \cdots \mathrm{H} 12^{\text {viii }}$ & 3.0700 \\
\hline $\mathrm{N} 1 \cdots \mathrm{H} 1$ & 1.8400 & $\mathrm{C} 12 \cdots \mathrm{H} 11^{\mathrm{viii}}$ & 2.8800 \\
\hline $\mathrm{C} 1 \cdots \mathrm{C} 3^{\text {iv }}$ & $3.594(3)$ & $\mathrm{C} 12 \cdots \mathrm{H} 12^{\text {viii }}$ & 3.0400 \\
\hline $\mathrm{C} 1 \cdots \mathrm{C}^{\text {iv }}$ & $3.448(3)$ & $\mathrm{C} 13 \cdots \mathrm{H} 11^{\mathrm{viii}}$ & 3.0600 \\
\hline $\mathrm{C} 1 \cdots \mathrm{C} 7^{\mathrm{v}}$ & $3.599(3)$ & $\mathrm{C} 15 \cdots \mathrm{H} 2$ & 2.6100 \\
\hline $\mathrm{C} 1 \cdots \mathrm{C} 8^{\mathrm{v}}$ & $3.320(3)$ & $\mathrm{H} 1 \cdots \mathrm{N} 1$ & 1.8400 \\
\hline $\mathrm{C} 1 \cdots \mathrm{C} 9^{\mathrm{v}}$ & $3.561(3)$ & $\mathrm{H} 1 \cdots \mathrm{C} 1$ & 3.0600 \\
\hline
\end{tabular}




\begin{tabular}{|c|c|c|c|}
\hline $\mathrm{C} 2 \cdots \mathrm{C} 9^{v}$ & $3.572(3)$ & $\mathrm{H} 1 \cdots \mathrm{C} 7$ & 2.4100 \\
\hline $\mathrm{C} 2 \cdots \mathrm{C} 14^{\text {iv }}$ & $3.547(3)$ & $\mathrm{H} 2 \cdots \mathrm{C} 15$ & 2.6100 \\
\hline $\mathrm{C} 3 \cdots \mathrm{N} 1^{\mathrm{v}}$ & $3.292(3)$ & $\mathrm{H} 2 \cdots \mathrm{H} 15 \mathrm{~A}$ & 1.8800 \\
\hline $\mathrm{C} 3 \cdots \mathrm{C} 1^{\mathrm{v}}$ & $3.594(3)$ & $\mathrm{H} 4 \cdots \mathrm{O} 2$ & 2.5200 \\
\hline $\mathrm{C} 4 \cdots \mathrm{C}^{\mathrm{v}}$ & $3.448(3)$ & $\mathrm{H} 4 \cdots \mathrm{H} 12^{\mathrm{x}}$ & 2.6000 \\
\hline $\mathrm{C} 5 \cdots \mathrm{C}^{\mathrm{v}}$ & $3.563(3)$ & $\mathrm{H} 5 \cdots \mathrm{O} 1^{\mathrm{xi}}$ & 2.7600 \\
\hline $\mathrm{C} 6 \cdots \mathrm{C} 7^{\mathrm{v}}$ & $3.542(3)$ & $\mathrm{H} 5 \cdots \mathrm{C} 9^{\mathrm{xi}}$ & 2.9700 \\
\hline $\mathrm{C} 7 \cdots \mathrm{C} 6^{\mathrm{iv}}$ & $3.542(3)$ & $\mathrm{H} 5 \cdots \mathrm{C} 10^{\mathrm{xi}}$ & 2.8900 \\
\hline $\mathrm{C} 7 \cdots \mathrm{C} 5^{\text {iv }}$ & $3.563(3)$ & $\mathrm{H} 6 \cdots \mathrm{C} 7$ & 2.6500 \\
\hline $\mathrm{C} 7 \cdots \mathrm{C} 11^{\mathrm{v}}$ & $3.485(3)$ & $\mathrm{H} 6 \cdots \mathrm{H} 7$ & 2.0300 \\
\hline $\mathrm{C} 7 \cdots \mathrm{C} 13^{v}$ & $3.536(3)$ & $\mathrm{H} 6 \cdots \mathrm{O} 2^{\mathrm{vi}}$ & 2.6900 \\
\hline $\mathrm{C} 7 \cdots^{\cdots} \mathrm{C} 12^{v}$ & $3.278(3)$ & $\mathrm{H} 7 \cdots \mathrm{C} 6$ & 2.5600 \\
\hline $\mathrm{C} 7 \cdots \mathrm{O} 2^{\mathrm{vi}}$ & $3.321(3)$ & $\mathrm{H} 7 \cdots \mathrm{H} 6$ & 2.0300 \\
\hline $\mathrm{C} 7 \cdots \mathrm{C} 1^{\text {iv }}$ & $3.599(3)$ & $\mathrm{H} 7 \cdots \mathrm{H} 13$ & 2.4500 \\
\hline $\mathrm{C} 8 \cdots \mathrm{C} 11^{\mathrm{v}}$ & $3.465(3)$ & $\mathrm{H} 7 \cdots \mathrm{O} 2^{\mathrm{vi}}$ & 2.4000 \\
\hline $\mathrm{C} 8 \cdots \mathrm{C} 1^{\text {iv }}$ & $3.320(3)$ & $\mathrm{H} 10 \cdots \mathrm{C}^{\mathrm{xii}}$ & 3.0200 \\
\hline $\mathrm{C} 8{ }^{\cdots} \mathrm{C} 12^{\mathrm{v}}$ & $3.563(3)$ & $\mathrm{H} 10 \cdots \mathrm{C} 6^{\mathrm{xii}}$ & 2.9800 \\
\hline $\mathrm{C} 9 \cdots \mathrm{C} 2^{\mathrm{iv}}$ & $3.572(3)$ & $\mathrm{H} 10 \cdots \mathrm{H} 15 \mathrm{~B}^{\mathrm{xiii}}$ & 2.6000 \\
\hline C $9 \cdots \mathrm{C} 11^{\mathrm{v}}$ & $3.591(3)$ & $\mathrm{H} 11 \cdots \mathrm{C} 5^{\mathrm{xii}}$ & 2.9900 \\
\hline $\mathrm{C} 9 \cdots \mathrm{C} 1^{\text {iv }}$ & $3.561(3)$ & $\mathrm{H} 11 \cdots \mathrm{C} 12^{\mathrm{vii}}$ & 2.8800 \\
\hline $\mathrm{C} 11 \cdots \mathrm{C} 8^{\mathrm{iv}}$ & $3.465(3)$ & $\mathrm{H} 11 \cdots \mathrm{C} 13^{\text {vii }}$ & 3.0600 \\
\hline $\mathrm{C} 11 \cdots \mathrm{C} 7^{\mathrm{iv}}$ & $3.485(3)$ & $\mathrm{H} 12 \cdots \mathrm{C} 11^{\mathrm{vii}}$ & 3.0700 \\
\hline $\mathrm{C} 11 \cdots \mathrm{C} 12^{\mathrm{vii}}$ & $3.565(3)$ & $\mathrm{H} 12 \cdots \mathrm{C} 12^{\mathrm{vii}}$ & 3.0400 \\
\hline $\mathrm{C} 11 \cdots \mathrm{C} 9^{\text {iv }}$ & $3.591(3)$ & $\mathrm{H} 12 \cdots \mathrm{H} 4^{\text {xiv }}$ & 2.6000 \\
\hline $\mathrm{C} 12 \cdots \mathrm{C} 11^{\text {viii }}$ & $3.565(3)$ & $\mathrm{H} 13 \cdots \mathrm{H} 7$ & 2.4500 \\
\hline $\mathrm{C} 12 \cdots \mathrm{C} 7^{\text {iv }}$ & $3.278(3)$ & $\mathrm{H} 15 \mathrm{~A} \cdots \mathrm{C} 2$ & 2.4700 \\
\hline $\mathrm{C} 12 \cdots \mathrm{C} 8^{\text {iv }}$ & $3.563(3)$ & $\mathrm{H} 15 \mathrm{~A} \cdots \mathrm{H} 2$ & 1.8800 \\
\hline $\mathrm{C} 13 \cdots \mathrm{C} 7^{\mathrm{iv}}$ & $3.536(3)$ & $\mathrm{H} 15 \mathrm{~A} \cdots \mathrm{H} 15 \mathrm{C}^{\mathrm{ii}}$ & 2.4600 \\
\hline $\mathrm{C} 14 \cdots \mathrm{C} 2^{\mathrm{v}}$ & $3.547(3)$ & $\mathrm{H} 15 \mathrm{~B} \cdots \mathrm{O} 1^{\mathrm{xv}}$ & 2.7200 \\
\hline $\mathrm{C} 1 \cdots \mathrm{H} 1$ & 3.0600 & $\mathrm{H} 15 \mathrm{~B} \cdots \mathrm{H} 10^{\mathrm{xvi}}$ & 2.6000 \\
\hline $\mathrm{C} 2 \cdots \mathrm{H} 15 \mathrm{~A}$ & 2.4700 & $\mathrm{H} 15 \mathrm{C} \cdots \mathrm{H} 15 \mathrm{~A}^{\mathrm{xv}}$ & 2.4600 \\
\hline $\mathrm{C} 9-\mathrm{O} 1-\mathrm{H} 1$ & 109.00 & $\mathrm{O} 2-\mathrm{C} 14-\mathrm{C} 3$ & $120.5(2)$ \\
\hline $\mathrm{C} 1-\mathrm{N} 1-\mathrm{C} 7$ & $121.25(18)$ & $\mathrm{C} 1-\mathrm{C} 2-\mathrm{H} 2$ & 120.00 \\
\hline $\mathrm{N} 1-\mathrm{C} 1-\mathrm{C} 2$ & $115.86(19)$ & $\mathrm{C} 3-\mathrm{C} 2-\mathrm{H} 2$ & 120.00 \\
\hline $\mathrm{N} 1-\mathrm{C} 1-\mathrm{C} 6$ & $125.3(2)$ & $\mathrm{C} 3-\mathrm{C} 4-\mathrm{H} 4$ & 120.00 \\
\hline $\mathrm{C} 2-\mathrm{C} 1-\mathrm{C} 6$ & $118.9(2)$ & $\mathrm{C} 5-\mathrm{C} 4-\mathrm{H} 4$ & 120.00 \\
\hline $\mathrm{C} 1-\mathrm{C} 2-\mathrm{C} 3$ & $120.8(2)$ & $\mathrm{C} 4-\mathrm{C} 5-\mathrm{H} 5$ & 120.00 \\
\hline $\mathrm{C} 2-\mathrm{C} 3-\mathrm{C} 4$ & $119.9(2)$ & $\mathrm{C} 6-\mathrm{C} 5-\mathrm{H} 5$ & 120.00 \\
\hline $\mathrm{C} 2-\mathrm{C} 3-\mathrm{C} 14$ & $121.4(2)$ & $\mathrm{C} 1-\mathrm{C} 6-\mathrm{H} 6$ & 120.00 \\
\hline $\mathrm{C} 4-\mathrm{C} 3-\mathrm{C} 14$ & $118.7(2)$ & $\mathrm{C} 5-\mathrm{C} 6-\mathrm{H} 6$ & 120.00 \\
\hline $\mathrm{C} 3-\mathrm{C} 4-\mathrm{C} 5$ & $119.4(2)$ & $\mathrm{N} 1-\mathrm{C} 7-\mathrm{H} 7$ & 120.00 \\
\hline $\mathrm{C} 4-\mathrm{C} 5-\mathrm{C} 6$ & $120.5(2)$ & $\mathrm{C} 8-\mathrm{C} 7-\mathrm{H} 7$ & 120.00 \\
\hline $\mathrm{C} 1-\mathrm{C} 6-\mathrm{C} 5$ & $120.5(2)$ & $\mathrm{C} 9-\mathrm{C} 10-\mathrm{H} 10$ & 120.00 \\
\hline $\mathrm{N} 1-\mathrm{C} 7-\mathrm{C} 8$ & $120.8(2)$ & $\mathrm{C} 11-\mathrm{C} 10-\mathrm{H} 10$ & 120.00 \\
\hline $\mathrm{C} 7-\mathrm{C} 8-\mathrm{C} 13$ & $119.6(2)$ & $\mathrm{C} 10-\mathrm{C} 11-\mathrm{H} 11$ & 120.00 \\
\hline $\mathrm{C} 9-\mathrm{C} 8-\mathrm{C} 13$ & $118.7(2)$ & $\mathrm{C} 12-\mathrm{C} 11-\mathrm{H} 11$ & 120.00 \\
\hline $\mathrm{C} 7-\mathrm{C} 8-\mathrm{C} 9$ & $121.7(2)$ & $\mathrm{C} 11-\mathrm{C} 12-\mathrm{H} 12$ & 120.00 \\
\hline
\end{tabular}




$\begin{array}{llll}\mathrm{O} 1-\mathrm{C} 9-\mathrm{C} 10 & 119.10(19) & \mathrm{C} 13-\mathrm{C} 12-\mathrm{H} 12 & 120.00 \\ \mathrm{C} 8-\mathrm{C} 9-\mathrm{C} 10 & 119.6(2) & \mathrm{C} 8-\mathrm{C} 13-\mathrm{H} 13 & 119.00 \\ \mathrm{O} 1-\mathrm{C} 9-\mathrm{C} 8 & 121.3(2) & \mathrm{C} 12-\mathrm{C} 13-\mathrm{H} 13 & 119.00 \\ \mathrm{C} 9-\mathrm{C} 10-\mathrm{C} 11 & 120.3(2) & \mathrm{C} 14-\mathrm{C} 15-\mathrm{H} 15 \mathrm{~A} & 109.00 \\ \mathrm{C} 10-\mathrm{C} 11-\mathrm{C} 12 & 120.8(2) & \mathrm{C} 14-\mathrm{C} 15-\mathrm{H} 15 \mathrm{~B} & 109.00 \\ \mathrm{C} 11-\mathrm{C} 12-\mathrm{C} 13 & 119.5(2) & \mathrm{C} 14-\mathrm{C} 15-\mathrm{H} 15 \mathrm{C} & 109.00 \\ \mathrm{C} 8-\mathrm{C} 13-\mathrm{C} 12 & 121.1(2) & \mathrm{H} 15 \mathrm{~A}-\mathrm{C} 15-\mathrm{H} 15 \mathrm{~B} & 109.00 \\ \mathrm{O} 2-\mathrm{C} 14-\mathrm{C} 15 & 120.9(2) & \mathrm{H} 15 \mathrm{~A}-\mathrm{C} 15-\mathrm{H} 15 \mathrm{C} & 109.00 \\ \mathrm{C} 3-\mathrm{C} 14-\mathrm{C} 15 & 118.5(2) & \mathrm{H} 15 \mathrm{~B}-\mathrm{C} 15-\mathrm{H} 15 \mathrm{C} & 109.00 \\ & & & \\ \mathrm{C} 7-\mathrm{N} 1-\mathrm{C} 1-\mathrm{C} 2 & -177.1(2) & \mathrm{C} 3-\mathrm{C} 4-\mathrm{C} 5-\mathrm{C} 6 & 1.8(4) \\ \mathrm{C} 7-\mathrm{N} 1-\mathrm{C} 1-\mathrm{C} 6 & 3.0(3) & \mathrm{C} 4-\mathrm{C} 5-\mathrm{C} 6-\mathrm{C} 1 & -1.0(4) \\ \mathrm{C} 1-\mathrm{N} 1-\mathrm{C} 7-\mathrm{C} 8 & -179.4(2) & \mathrm{N} 1-\mathrm{C} 7-\mathrm{C} 8-\mathrm{C} 9 & -1.3(3) \\ \mathrm{N} 1-\mathrm{C} 1-\mathrm{C} 2-\mathrm{C} 3 & 179.9(2) & \mathrm{N} 1-\mathrm{C} 7-\mathrm{C} 8-\mathrm{C} 13 & 178.4(2) \\ \mathrm{C} 6-\mathrm{C} 1-\mathrm{C} 2-\mathrm{C} 3 & -0.2(3) & \mathrm{C} 7-\mathrm{C} 8-\mathrm{C} 9-\mathrm{O} 1 & 0.1(3) \\ \mathrm{N} 1-\mathrm{C} 1-\mathrm{C} 6-\mathrm{C} 5 & -179.9(2) & \mathrm{C} 7-\mathrm{C} 8-\mathrm{C} 9-\mathrm{C} 10 & -179.7(2) \\ \mathrm{C} 2-\mathrm{C} 1-\mathrm{C} 6-\mathrm{C} 5 & 0.1(4) & \mathrm{C} 13-\mathrm{C} 8-\mathrm{C} 9-\mathrm{O} 1 & -179.6(2) \\ \mathrm{C} 1-\mathrm{C} 2-\mathrm{C} 3-\mathrm{C} 4 & 1.0(4) & \mathrm{C} 13-\mathrm{C} 8-\mathrm{C} 9-\mathrm{C} 10 & 0.7(3) \\ \mathrm{C} 1-\mathrm{C} 2-\mathrm{C} 3-\mathrm{C} 14 & -178.4(2) & \mathrm{C} 7-\mathrm{C} 8-\mathrm{C} 13-\mathrm{C} 12 & -179.7(2) \\ \mathrm{C} 2-\mathrm{C} 3-\mathrm{C} 4-\mathrm{C} 5 & -1.8(3) & \mathrm{C} 9-\mathrm{C} 8-\mathrm{C} 13-\mathrm{C} 12 & -0.1(3) \\ \mathrm{C} 14-\mathrm{C} 3-\mathrm{C} 4-\mathrm{C} 5 & 177.6(2) & \mathrm{O} 1-\mathrm{C} 9-\mathrm{C} 10-\mathrm{C} 11 & 180.0(2) \\ \mathrm{C} 2-\mathrm{C} 3-\mathrm{C} 14-\mathrm{O} 2 & -171.1(2) & \mathrm{C} 8-\mathrm{C} 9-\mathrm{C} 10-\mathrm{C} 11 & -0.3(3) \\ \mathrm{C} 2-\mathrm{C} 3-\mathrm{C} 14-\mathrm{C} 15 & 11.1(3) & \mathrm{C} 9-\mathrm{C} 10-\mathrm{C} 11-\mathrm{C} 12 & -0.7(3) \\ \mathrm{C} 4-\mathrm{C} 3-\mathrm{C} 14-\mathrm{O} 2 & 9.5(4) & \mathrm{C} 10-\mathrm{C} 11-\mathrm{C} 12-\mathrm{C} 13 & 1.3(4) \\ \mathrm{C} 4-\mathrm{C} 3-\mathrm{C} 14-\mathrm{C} 15 & -168.4(2) & \mathrm{C} 11-\mathrm{C} 12-\mathrm{C} 13-\mathrm{C} 8 & -0.9(3) \\ & & & \end{array}$

Symmetry codes: (i) $-x+1, y+1 / 2,-z+1 / 2$; (ii) $x-1 / 2,-y+1 / 2,-z+1$; (iii) $-x+1 / 2,-y, z+1 / 2$; (iv) $x-1, y, z$; (v) $x+1, y, z$; (vi) $-x+1, y-1 / 2,-z+1 / 2$; (vii) $x-1 / 2,-y-1 / 2,-z+1$; (viii) $x+1 / 2,-y-1 / 2,-z+1$; (ix) $-x-1 / 2,-y, z-1 / 2$; (x) $-x, y+1 / 2,-z+1 / 2$; (xi) $-x+1 / 2,-y, z-1 / 2$; (xii) $-x-1 / 2,-y, z+1 / 2$; (xiii) $x-3 / 2$, $-y+1 / 2,-z+1$; (xiv) $-x, y-1 / 2,-z+1 / 2$; (xv) $x+1 / 2,-y+1 / 2,-z+1$; (xvi) $x+3 / 2,-y+1 / 2,-z+1$.

Hydrogen-bond geometry $\left(\AA,{ }^{\circ}\right)$

\begin{tabular}{lllll}
\hline$D-\mathrm{H}^{\prime} \cdots A$ & $D-\mathrm{H}$ & $\mathrm{H} \cdots A$ & $D \cdots A$ & $D-\mathrm{H} \cdots A$ \\
\hline $\mathrm{O} 1-\mathrm{H} 1 \cdots \mathrm{N} 1$ & 0.84 & 1.84 & $2.590(2)$ & 148 \\
$\mathrm{C} 7-\mathrm{H} 7 \cdots \mathrm{O} 2^{\text {vi }}$ & 0.95 & 2.40 & $3.321(3)$ & 162 \\
\hline
\end{tabular}

Symmetry code: (vi) $-x+1, y-1 / 2,-z+1 / 2$. 